TRANSACTIONS OF THE

AMERICAN MATHEMATICAL SOCIETY

Volume 357, Number 5, Pages 2021-2042

S 0002-9947(04)03683-9

Article electronically published on November 4, 2004

\title{
MANSFIELD'S IMPRIMITIVITY THEOREM FOR FULL CROSSED PRODUCTS
}

\author{
S. KALISZEWSKI AND JOHN QUIGG
}

\begin{abstract}
For any maximal coaction $(A, G, \delta)$ and any closed normal subgroup $N$ of $G$, there exists an imprimitivity bimodule $Y_{G / N}^{G}(A)$ between the full crossed product $A \times_{\delta} G \times \times_{\widehat{\delta} \mid} N$ and $A \times{ }_{\delta \mid} G / N$, together with $\operatorname{Inf} \widehat{\widehat{\delta}} \mid-\delta^{\text {dec }}$ compatible coaction $\delta_{Y}$ of $G$. The assignment $(A, \delta) \mapsto\left(Y_{G / N}^{G}(A), \delta_{Y}\right)$ implements a natural equivalence between the crossed-product functors " $\times G \times N$ " and " $\times G / N$ ", in the category whose objects are maximal coactions of $G$ and whose morphisms are isomorphism classes of right-Hilbert bimodule coactions of $G$.
\end{abstract}

\section{INTRODUCTION}

For an action $\alpha$ of a locally compact group $G$ on a $C^{*}$-algebra $B$ and a closed normal subgroup $N$ of $G$, Green's imprimitivity theorem (as formulated, for example, in [4, Theorem B.5]) says that the iterated crossed product $B \times{ }_{\alpha} G \times{ }_{\widehat{\alpha} \mid} G / N$ by the restriction of the dual coaction $\widehat{\alpha}$ is Morita-Rieffel equivalent to $B \times_{\alpha \mid} N$. Thus, a representation of $B \times{ }_{\alpha} G$ is induced from a representation of $B \times{ }_{\alpha \mid} N$ if and only if it can be combined with a representation of $C_{0}(G / N)$ to make a covariant representation of the system $\left(B \times{ }_{\alpha} G, G / N, \widehat{\alpha} \mid\right)$. This represents the culmination of roughly 50 years of imprimitivity theorems, dating back to Mackey [10].

Green's theorem starts with an action, and uses a dual coaction; for symmetry one desires a "dual" version which starts with a coaction and uses a dual action. In 1991 Mansfield [1] gave the first such result: if $\delta$ is a reduced coaction of $G$ on a $C^{*}$-algebra $A$, and if the normal subgroup $N$ is amenable, then $A \times{ }_{\delta} G \times{ }_{\widehat{\delta} \mid} N$ is Morita-Rieffel equivalent to $A \times_{\delta \mid} G / N$. This theorem suffers from two significant drawbacks: the coaction is reduced, meaning it uses the reduced algebra $C_{r}^{*}(G)$ and the theory is decidedly spatial; and the subgroup $N$ is required to be amenable, in order to have a surjection $C_{r}^{*}(G) \rightarrow C_{r}^{*}(G / N)$ which vouchsafes the restricted coaction $\delta \mid$. In 9] we removed these drawbacks by using full coactions, meaning the full group algebra $C^{*}(G)$ is used and the theory is nonspatial; consequently we no longer needed $N$ to be amenable. However, the price was that we then had to require our coactions to be normal (an unfortunate term meaning essentially that crossed product duality holds for reduced crossed products) and use the reduced crossed by the dual action.

Received by the editors December 12, 2003.

2000 Mathematics Subject Classification. Primary 46L55.

Key words and phrases. $C^{*}$-algebra, locally compact group, coaction, right-Hilbert bimodule, duality, naturality.

(C)2004 American Mathematical Society 
This is in some sense a ridiculous situation; the whole point of crossed products is to encode all the covariant representations, not just those that factor through the regular representation. What is of course desired is to use full crossed products throughout. The first result in this vein was given in 1998 by Echterhoff, Raeburn, and the first author [5]: if $\delta$ is a dual coaction, then $A \times{ }_{\delta} G \times{ }_{\delta \mid} N$ is Morita-Rieffel equivalent to $A \times_{\delta \mid} G / N$, where all crossed products are now full. Relaxing the restriction that $\delta$ be dual was partially accomplished by Echterhoff and the second author [6], but only for discrete groups. The missing ingredient was a crossed product duality for full crossed products. With Echterhoff, we recently introduced maximal coactions [2], which are by definition those for which full crossed product duality holds; dual coactions and discrete coactions are specific examples. The term maximal was chosen because for every coaction $(A, G, \delta)$ there is a maximal coaction $\delta^{m}$ on an extension $A^{m}$ of $A$ such that $A^{m} \times{ }_{\delta^{m}} G \cong A \times{ }_{\delta} G$. By contrast, there is also a normal coaction $\delta^{n}$ on a quotient $A^{n}$ of $A$ with the same crossed product 14] (and in retrospect perhaps a better term for normal coactions might be minimal).

In this paper we prove Mansfield's imprimitivity theorem for full crossed products (Theorem [5.3): for every maximal coaction $(A, G, \delta)$ and any closed normal subgroup $N$ of $G$, the full crossed product $A \times{ }_{\delta} G \times \times_{\widehat{\delta} \mid} N$ is Morita-Rieffel equivalent to $A \times_{\delta \mid} G / N$. We also prove that this equivalence is equivariant for the appropriate coactions of $G$. As mentioned above, an important ingredient in the proof is a full crossed-product duality theorem for maximal coactions, which allows us to switch from an arbitrary maximal coaction to a dual coaction. The bimodule involved, which we call the Katayama bimodule, is also of interest for nonmaximal coactions, and we investigate it in detail in Section 4 .

We also fit our version of Mansfield's imprimitivity theorem into a categorical perspective that we, along with Echterhoff and Raeburn 3, 4, have been pushing: there is a category whose objects are $C^{*}$-algebras and whose morphisms are isomorphism classes of right-Hilbert bimodules (see Section 2 for a more detailed summary), and for a fixed group $G$ there are equivariant versions of this category which incorporate actions and coactions of $G$. Crossed products are functors among these categories, and imprimitivity theorems express natural equivalences between them. The naturality theorem for full Mansfield imprimitivity is Theorem6.6, Our proof exploits a curious relationship (Corollary 6.4) between Green's imprimitivity bimodule and the Katayama bimodule; naturality of those bimodules is proved in Sections 3 and 4 , respectively.

In Section 7 we prove that maximality is preserved under various operations: restriction to quotients, inflation from subgroups, decomposition, and restriction to invariant ideals. Besides being of obvious interest and technical utility, the results in this section allow us to say that the functors in the naturality-of-Mansfield theorem actually live completely within the category of maximal coactions.

\section{Preliminaries}

We adopt the conventions of [4] for almost everything. However, in some aspects our conventions are slightly different from other sources, so to avoid any confusion we summarize the possibly unfamiliar details here. 
2.1. Right-Hilbert bimodules. Let $A$ and $B$ be $C^{*}$-algebras. A right-Hilbert $A-B$ bimodule, sometimes denoted ${ }_{A} X_{B}$, is a (right) Hilbert $B$-module $X$ with a compatible, nondegenerate, left $A$-action. We do not require the $B$-valued inner product to be full, that is, the closed ideal

$$
B_{X}:=\overline{\langle X, X\rangle_{B}}
$$

of $B$ is allowed to be proper. Compatibility of the left $A$-action means that $X$ is an $A-B$ bimodule in the usual algebraic sense, that is, $(a \cdot x) \cdot b=a \cdot(x \cdot b)$ for all $a \in A, x \in X$, and $b \in B$. Nondegeneracy can be interpreted in two equivalent ways: the closed span $\overline{A X}$ coincides with $X$, or the associated homomorphism $\kappa_{A}: A \rightarrow M(\mathcal{K}(X))$ is nondegenerate, where $\mathcal{K}(X)$ is the imprimitivity algebra of the Hilbert $B$-module $X$.

A right-Hilbert bimodule ${ }_{A} X_{B}$ is full if it is full as a Hilbert $B$-module, that is, if $B_{X}=B$. By restricting the right-hand coefficient algebra, every right Hilbert $A-B$ bimodule $X$ may be regarded as a full right-Hilbert $A-B_{X}$ bimodule. Also, we may regard $X$ as a right-Hilbert $\mathcal{K}(X)-B$ bimodule.

If $\phi: A \rightarrow M(B)$ is a homomorphism, the closed span $\overline{\phi(A) B}$ is called a standard right-Hilbert $A-B$ bimodule. If $\phi$ is nondegenerate, that is, if $\overline{\phi(A) B}=B$, then $B$ is called a nondegenerate standard right-Hilbert bimodule. If ${ }_{A} X_{B}$ is any rightHilbert bimodule, then $\kappa_{A}$ makes $\mathcal{K}(X)$ into a nondegenerate standard $A-\mathcal{K}(X)$ bimodule.

A partial $A-B$ imprimitivity bimodule is a right-Hilbert bimodule ${ }_{A} X_{B}$ which is also a left Hilbert $A$-module such that the inner products satisfy the compatibility condition

$$
{ }_{A}\langle x, y\rangle z=x\langle y, z\rangle_{B}
$$

for all $x, y, z \in X$. Of course, if both inner products are full, then $X$ is an imprimitivity bimodule in the usual sense. A right-partial imprimitivity bimodule is a partial imprimitivity bimodule whose left inner product is full (and similarly for left-partial). Every right-Hilbert $A-B$ bimodule may be regarded as a $\mathcal{K}(X)-B_{X}$ imprimitivity bimodule, or alternatively as a right-partial $\mathcal{K}(X)-B$ imprimitivity bimodule.

Every partial $A-B$ imprimitivity bimodule $X$ has a linking algebra $L(X)$, which is a $C^{*}$-algebra characterized by the existence of complementary projections $p, q \in M(L(X))$ such that

$$
A=p L(X) p, \quad B=q L(X) q, \quad \text { and } \quad X=p L(X) q .
$$

Of course, the projections $p$ and $q$ are full if and only if $X$ is actually an $A-B$ imprimitivity bimodule.

The multiplier bimodule of a right-Hilbert $A-B$ bimodule $X$ is the space $M(X)$ of maps $T: B \rightarrow X$ which are adjointable in the sense that there exists $T^{*}: X \rightarrow B$ with

$$
\langle T b, x\rangle_{B}=b^{*} T^{*} x
$$

for all $x \in X$ and $b \in B$. (Such maps are automatically bounded and $B$-linear.) $M(X)$ is a right-Hilbert $M(A)-M(B)$ bimodule in a natural way.

If ${ }_{A} X_{B}$ and ${ }_{C} Y_{D}$ are right-Hilbert bimodules and $\phi: A \rightarrow M(C)$ and $\psi: B \rightarrow$ $M(D)$ are homomorphisms, a $\phi-\psi$ compatible right-Hilbert bimodule homomorphism is a linear map $\Phi: X \rightarrow M(Y)$ such that

$\Phi(a \cdot x)=\phi(a) \cdot \Phi(x), \quad \Phi(x \cdot b)=\Phi(x) \cdot \psi(b), \quad$ and $\quad\langle\Phi(x), \Phi(z)\rangle_{M(D)}=\psi\left(\langle x, z\rangle_{B}\right)$ 
for all $a \in A, x, z \in X$, and $b \in B$. $\Phi$ is nondegenerate if $\overline{\Phi(X) D}=Y$ and the coefficient maps $\phi$ and $\psi$ are nondegenerate.

An isomorphism of right-Hilbert $A-B$ bimodules $X$ and $Y$ is linear bijection $\Phi: X \rightarrow Y$ which is an $\operatorname{id}_{A}-\operatorname{id}_{B}$ compatible right-Hilbert bimodule homomorphism. For the homomorphism property, it suffices to check the first and third conditions above, since then $\Phi$ automatically preserves the right $B$-action.

2.2. The basic category. Our basic category $\mathcal{C}$ has $C^{*}$-algebras as objects, and the morphisms from an object $A$ to an object $B$ are the isomorphism classes of right-Hilbert $A-B$ bimodules. (We ignore the nuisance of $\operatorname{Mor}(A, B)$ not being a set - this never causes problems in practice; more precisely, in any given situation of interest the choice of morphisms can be suitably restricted.)

Given right-Hilbert bimodules ${ }_{A} X_{B}$ and ${ }_{B} Y_{C}$, the balanced tensor product $X \otimes_{B}$ $Y$ is a right Hilbert $A-C$ bimodule. The composition $[Y] \circ[X]$ of the morphisms $[X]$ and $[Y]$ in the category $\mathcal{C}$ is the isomorphism class $\left[X \otimes_{B} Y\right]$. We often express the fact that ${ }_{A} Z_{C} \cong{ }_{A} X \otimes_{B} Y_{C}$ by declaring that the diagram

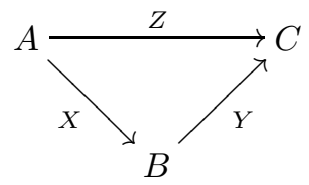

commutes. The identity morphism on an object $A$ is the isomorphism class of the standard right-Hilbert bimodule ${ }_{A} A_{A}$. The equivalences (that is, the invertible morphisms) in $\mathcal{C}$ are exactly the isomorphism classes of imprimitivity bimodules.

Every morphism in $\mathcal{C}$ can be factored as a composition of a nondegenerate standard morphism and a right-partial equivalence: namely,

$$
{ }_{A} X_{B} \cong{ }_{A} \mathcal{K}(X) \otimes_{\mathcal{K}(X)} X_{B},
$$

so $\left[{ }_{A} X_{B}\right]=\left[{ }_{\mathcal{K}(X)} X_{B}\right] \circ\left[{ }_{A} \mathcal{K}(X)_{\mathcal{K}(X)}\right]$.

2.3. Equivariant categories. Given a right-Hilbert bimodule ${ }_{A} X_{B}$ and actions $(A, \alpha)$ and $(B, \beta)$ of a locally compact group $G$, an $\alpha-\beta$ compatible action on $X$ is a point-norm continuous map $\gamma$ of $G$ into the linear bijections on $X$ such that

$$
\gamma_{s}(a x)=\alpha_{s}(a) \gamma_{s}(x) \text { and }\left\langle\gamma_{s}(x), \gamma_{s}(y)\right\rangle_{B}=\beta_{s}\left(\langle x, y\rangle_{B}\right)
$$

for all $s \in G, a \in A$, and $x, y \in X$. (The property $\gamma_{s}(x b)=\gamma_{s}(x) \beta_{s}(b)$ for all $s \in G$, $x \in X$, and $b \in B$ is then automatic.)

Given another action $(C, \epsilon)$ and a $\beta-\epsilon$ compatible right-Hilbert bimodule action $(Y, \rho)$, the $\alpha-\epsilon$ compatible balanced tensor product action $\gamma \otimes_{B} \rho$ on $X \otimes_{B} Y$ is given by

$$
\left(\gamma \otimes_{B} \rho\right)_{s}(x \otimes y)=\gamma_{s}(x) \otimes \rho_{s}(y) .
$$

If $(X, \gamma)$ and $(Y, \rho)$ are $\alpha-\beta$ compatible actions, a right-Hilbert bimodule isomorphism $\Phi: X \rightarrow Y$ is $\gamma-\rho$ equivariant if it intertwines $\gamma$ and $\rho$, that is, if $\Phi \circ \gamma_{s}=\rho_{s} \circ \Phi$ for all $s \in G$.

The category $\mathcal{A}(G)$ of actions of $G$ has $C^{*}$-algebra actions as objects and isomorphism classes of right-Hilbert bimodule actions as morphisms. The composition is given by the balanced tensor product

$$
[(Y, \rho)] \circ[(X, \gamma)]=\left[\left(X \otimes_{B} Y, \gamma \otimes_{B} \rho\right)\right]
$$


and the identity morphism on an object $(A, \alpha)$ is the isomorphism class of the standard bimodule action ${ }_{(A, \alpha)}(A, \alpha)_{(A, \alpha)}$.

Turning to the dual category, we must first issue a warning:

Warning. We assume without further comment that all $C^{*}$-coactions $(A, G, \delta)$ satisfy the nondegeneracy condition

$$
\overline{\delta(A)\left(1 \otimes C^{*}(G)\right)}=A \otimes C^{*}(G) .
$$

It is still an open problem whether the above nondegeneracy condition is automatic, and if it should turn out that non-nondegenerate coactions exist, they will be of no interest in duality theory. Thus, we feel that the nondegeneracy condition should be included in the definition of coaction.

Given a right-Hilbert bimodule ${ }_{A} X_{B}$ and coactions $(A, \delta)$ and $(B, \epsilon)$ of $G$, a $\delta-\epsilon$ compatible coaction on $X$ is a nondegenerate $\delta-\epsilon$ compatible right-Hilbert bimodule homomorphism $\zeta: X \rightarrow M\left(X \otimes C^{*}(G)\right)$ which satisfies the nondegeneracy condition

$$
\overline{\left(1 \otimes C^{*}(G)\right) \zeta(X)}=X \otimes C^{*}(G)
$$

and the coaction identity

$$
(\zeta \otimes \mathrm{id}) \circ \zeta=\left(\mathrm{id} \otimes \delta_{G}\right) \circ \zeta
$$

Thus we have built nondegeneracy of $\zeta$, both as a bimodule homomorphism and as a coaction, into the definition. The companion nondegeneracy condition

$$
\overline{\zeta(X)\left(1 \otimes C^{*}(G)\right)}=X \otimes C^{*}(G)
$$

is then automatic.

Given another coaction $(C, \vartheta)$ and a $\epsilon-\vartheta$ compatible right-Hilbert bimodule coaction $(Y, \eta)$, the $\delta-\vartheta$ compatible balanced tensor product coaction $\zeta \sharp_{B} \eta$ on $X \otimes_{B} Y$ is given by

$$
\zeta \sharp_{B} \eta=\Theta \circ\left(\zeta \otimes_{B} \eta\right),
$$

where $\Theta:\left(X \otimes C^{*}(G)\right) \otimes_{B \otimes C^{*}(G)}\left(Y \otimes C^{*}(G)\right) \rightarrow\left(X \otimes_{B} Y\right) \otimes C^{*}(G)$ is the isomorphism given by

$$
\Theta((x \otimes c) \otimes(y \otimes d))=(x \otimes y) \otimes c d .
$$

If $(X, \zeta)$ and $(Y, \eta)$ are $\delta-\epsilon$ compatible coactions, a right-Hilbert bimodule isomorphism $\Phi: X \rightarrow Y$ is $\zeta-\eta$ equivariant if

$$
\eta \circ \Phi=(\Phi \otimes \mathrm{id}) \circ \zeta .
$$

The category $\mathcal{C}(G)$ of coactions of $G$ has $C^{*}$-algebra coactions as objects and isomorphism classes of right-Hilbert bimodule coactions as morphisms. The composition is given by the balanced tensor product

$$
[(Y, \eta)] \circ[(X, \zeta)]=\left[\left(X \otimes_{B} Y, \zeta \sharp_{B} \eta\right)\right],
$$

and the identity morphism on an object $(A, \delta)$ is the isomorphism class of the standard bimodule coaction ${ }_{(A, \delta)}(A, \delta)_{(A, \delta)}$.

The familiar operations of forming crossed products, inflating, restricting, and forming decomposition actions or coactions are functors among the equivariant categories. 


\section{Naturality of Green's Bimodule}

Recall that for any action $(B, G, \alpha)$ and any closed normal subgroup $N$ of $G$, Green's imprimitivity theorem gives a $B \times{ }_{\alpha} G \times \times_{\widehat{\alpha} \mid} G / N-B \times{ }_{\alpha \mid} N$ imprimitivity bimodule $X_{N}^{G}(B)$ which is a completion of $C_{c}(G, B)$ ([8; see also [4] Appendix B]). There is also a $\widehat{\alpha}^{\mathrm{dec}}-\operatorname{Inf} \widehat{\alpha} \mid$ compatible coaction $\delta_{X(B)}$ on $X_{N}^{G}(B)$ which maps $C_{c}(G, B)$ into $C_{c}\left(G, M^{\beta}\left(B \otimes C^{*}(G)\right)\right) \subset M\left(X_{N}^{G}(B) \otimes C^{*}(G)\right)$ by the formula

$$
\delta_{X(B)}(x)(t)=x(t) \otimes t
$$

[4, Section 4.4]. (The superscript $\beta$ means we require the functions to be continuous for the strict topology on the multipliers.)

In this section we prove that Green's bimodule is a coaction-equivariant natural equivalence between certain crossed product functors. Without the equivariance, this was proven in [3]. With equivariance, it was proven in [4, Theorem 4.20], but in the context of reduced crossed products, and there the construction was deduced from a more general one involving induced actions. Since we rely heavily on naturality of Green's bimodule for some of our main results, we feel it is best to give the details here. It also provides a good illustration of the techniques which are typical in proving naturality theorems.

Theorem 3.1 (Naturality of Green). Let ${ }_{(B, \alpha)}(X, \gamma)_{(C, \beta)}$ be a right-Hilbert bimodule action of a locally compact group $G$, and let $N$ be a closed normal subgroup of $G$. Then the diagram

$$
\begin{gathered}
\left(B \times{ }_{\alpha} G \times \times_{\widehat{\alpha} \mid} G / N, \widehat{\alpha}^{d e c}\right) \stackrel{\left(X_{N}^{G}(B), \delta_{X(B)}\right)}{\longrightarrow}\left(B \times{ }_{\alpha \mid} N, \operatorname{Inf} \widehat{\alpha} \mid\right) \\
\left(X \times G \times G / N, \widehat{\gamma}^{d e c}\right) \downarrow \\
\left(C \times \times_{\beta} G \times \times_{\widehat{\beta} \mid} G / N, \widehat{\beta}^{d e c}\right) \frac{\mid(X \times N, \operatorname{Inf} \widehat{\gamma} \mid)}{\left(X_{N}^{G}(C), \delta_{X(C)}\right)}\left(C \times_{\beta}|N, \operatorname{Inf} \widehat{\beta}|\right)
\end{gathered}
$$

commutes.

Proof. We follow the strategy employed in both 3 and [4]: any such $(X, \gamma)$ can be factored as a balanced tensor product of a nondegenerate standard bimodule action and a right-partial imprimitivity bimodule action. Once we know the commutativity for each of these two special cases, the result follows by chaining together the corresponding diagrams, using functoriality of the crossed product.

So, first assume $(X, \gamma)$ is of the form $(C, \beta)$, where we have a nondegenerate $\alpha-\beta$ equivariant homomorphism $\phi: B \rightarrow M(C)[$ The required diagram is the outer rectangle of the diagram

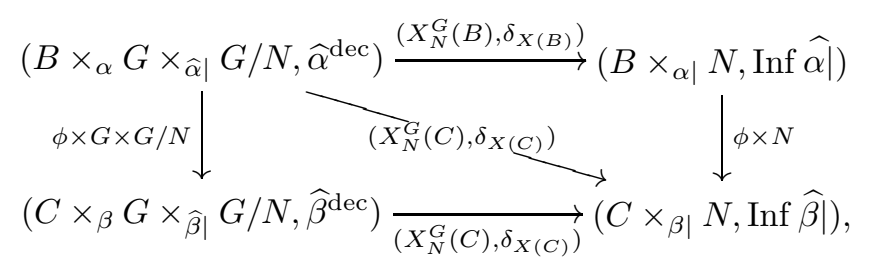

\footnotetext{
${ }^{1}$ For this part of the proof, by [4 Lemma 4.10] it suffices to find an equivariant imprimitivity bimodule homomorphism of $X_{N}^{G}(B)$ onto $X_{N}^{G}(C)$ whose coefficient maps are $\phi \times G \times G / N$ and $\phi \times N$. Instead, we will take advantage of the bimodule isomorphism already provided by 3 .
} 
where the bimodule on the diagonal is defined by the lower left triangle; the coaction on the diagonal is compatible with the appropriate coactions precisely because $\phi \times G \times G / N$ is $\widehat{\alpha}^{\mathrm{dec}}-\widehat{\beta}^{\mathrm{dec}}$ equivariant.

For the upper triangle, we use the right-Hilbert bimodule isomorphism $\Psi$ of $X_{N}^{G}(B) \otimes_{B \times N}\left(C \times_{\beta \mid} N\right)$ onto $X_{N}^{G}(C)$ provided by [3]. For an elementary tensor $x \otimes g \in C_{c}(G, B) \odot C_{c}(N, C), \Psi(x \otimes g) \in C_{c}(G, C)$ is defined by

$$
\Psi(x \otimes g)(t)=\int_{N} \phi(x(t n)) \beta_{t n}\left(g\left(n^{-1}\right)\right) \Delta(n)^{-1 / 2} d n .
$$

To see that $\Psi$ is $\delta_{X(B)} \sharp_{B \times N} \operatorname{Inf} \widehat{\beta} \mid-\delta_{X(C)}$ equivariant, compute

$$
\begin{aligned}
(\Psi & \otimes \mathrm{id}) \circ\left(\delta_{X(B)} \sharp \operatorname{Inf} \widehat{\beta} \mid\right)(x \otimes g)(t) \\
& =(\Psi \otimes \mathrm{id}) \circ \Theta\left(\delta_{X(B)}(x) \otimes \operatorname{Inf} \widehat{\beta} \mid(g)\right)(t) \\
& =\int(\phi \otimes \mathrm{id})\left(\delta_{X(B)}(x)(t n)\right)\left(\beta_{t n} \otimes \mathrm{id}\right)\left(\operatorname{Inf} \widehat{\beta} \mid(g)\left(n^{-1}\right)\right) \Delta(n)^{-1 / 2} d n \\
& =\int(\phi \otimes \mathrm{id})(x(t n) \otimes t n)\left(\beta_{t n} \otimes \mathrm{id}\right)\left(g\left(n^{-1}\right) \otimes n^{-1}\right) \Delta(n)^{-1 / 2} d n \\
& =\int(\phi(x(t n)) \otimes t n)\left(\beta_{t n}\left(g\left(n^{-1}\right)\right) \otimes n^{-1}\right) \Delta(n)^{-1 / 2} d n \\
& =\int\left(\phi(x(t n)) \beta_{t n}\left(g\left(n^{-1}\right)\right) \otimes t\right) \Delta(n)^{-1 / 2} d n \\
& =\int \phi(x(t n)) \beta_{t n}\left(g\left(n^{-1}\right)\right) \Delta(n)^{-1 / 2} d n \otimes t \\
& =\Psi(x \otimes g)(t) \otimes t \\
& =\delta_{X(C)} \circ \Psi(x \otimes g)(t) .
\end{aligned}
$$

The equality $\left(^{*}\right)$ is verified by temporarily replacing $\delta_{X(B)}(x)$ and $\operatorname{Inf} \widehat{\beta} \mid(g)$ by elementary tensors $x^{\prime} \otimes c \in C_{c}(G, B) \odot C^{*}(G)$ and $g^{\prime} \otimes d \in C_{c}(N, C) \odot C^{*}(G)$, computing that

$$
\begin{aligned}
(\Psi & \otimes \mathrm{id}) \circ \Theta\left(\left(x^{\prime} \otimes c\right) \otimes\left(g^{\prime} \otimes d\right)\right)(t) \\
& =(\Psi \otimes \mathrm{id})\left(\left(x^{\prime} \otimes g^{\prime}\right) \otimes c d\right)(t) \\
& =\left(\Psi\left(x^{\prime} \otimes g^{\prime}\right) \otimes c d\right)(t) \\
& =\Psi\left(x^{\prime} \otimes g^{\prime}\right)(t) \otimes c d \\
& =\int \phi\left(x^{\prime}(t n)\right) \beta_{t n}\left(g^{\prime}\left(n^{-1}\right)\right) \Delta(n)^{-1 / 2} d n \otimes c d \\
& =\int\left(\phi\left(x^{\prime}(t n)\right) \beta_{t n}\left(g^{\prime}\left(n^{-1}\right)\right) \otimes c d\right) \Delta(n)^{-1 / 2} d n \\
& =\int\left(\phi\left(x^{\prime}(t n)\right) \otimes c\right)\left(\beta_{t n}\left(g^{\prime}\left(n^{-1}\right)\right) \otimes d\right) \Delta(n)^{-1 / 2} d n \\
& =\int(\phi \otimes \mathrm{id})\left(x^{\prime}(t n) \otimes c\right)\left(\beta_{t n} \otimes \mathrm{id}\right)\left(g^{\prime}\left(n^{-1}\right) \otimes d\right) \Delta(n)^{-1 / 2} d n \\
& =\int(\phi \otimes \mathrm{id})\left(\left(x^{\prime} \otimes c\right)(t n)\right)\left(\beta_{t n} \otimes \mathrm{id}\right)\left(\left(g^{\prime} \otimes d\right)\left(n^{-1}\right)\right) \Delta(n)^{-1 / 2} d n
\end{aligned}
$$

and then appealing to density and continuity for the inductive-limit topologies. 
The argument for the lower triangle is much easier: if

$$
\Upsilon: C \times_{\beta} G \times \times_{\widehat{\beta} \mid} G / N \otimes_{C \times G \times G / N} X_{N}^{G}(C) \rightarrow X_{N}^{G}(C)
$$

is the canonical isomorphism, then for $f \in C \times{ }_{\beta} G \times \times_{\widehat{\beta} \mid} G / N$ and $x \in X_{N}^{G}(C)$ it is not hard to see that

$$
(\Upsilon \otimes \mathrm{id}) \circ\left(\widehat{\beta}^{\mathrm{dec}} \sharp_{C \times G \times G / N} \delta_{X(C)}\right)(f \otimes x)=\widehat{\beta}^{\mathrm{dec}}(f) \cdot \delta_{X(C)}(x),
$$

while

$$
\delta_{X(C)} \circ \Upsilon(f \otimes x)=\delta_{X(C)}(f \cdot x) ;
$$

so it amounts to the fact that $\delta_{X(C)}$ is a right-Hilbert bimodule homomorphism with left coefficient map $\widehat{\beta}^{\text {dec }}$.

Now assume that ${ }_{(B, \alpha)}(X, \gamma)_{(C, \beta)}$ is a right-partial imprimitivity bimodule action. Let $L(X)$ be the linking algebra of ${ }_{B} X_{C}$. Then there is an action $\nu$ on $L(X)$ which compresses on the corners to the given actions on $B, X$, and $C$. It follows straight from the definitions that the coaction $\delta_{X(L(X))}$ on the associated Green bimodule $X_{N}^{G}(L(X))$ compresses on the diagonal corners to $\delta_{X(B)}$ and $\delta_{X(C)}$. Using the identification

$$
L(X) \times{ }_{\nu} G \times \times_{\widehat{\nu} \mid} G / N=L\left(X \times_{\gamma} G \times \times_{\widehat{\gamma} \mid} G / N\right),
$$

we can apply the linking algebra techniques of 4 , Section 4.2$]$ to conclude that the desired diagram commutes, provided we can show

$$
\left\langle p X_{N}^{G}(L(X)) q, p X_{N}^{G}(L(X)) q\right\rangle_{C \times N} \subset \overline{\langle X \times N, X \times N\rangle_{C \times N}} .
$$

But we have

$$
\begin{aligned}
\left\langle p C_{c}(G, L(X)) q, p C_{c}(G, L(X)) q\right\rangle_{C \times N} & =\left\langle C_{c}(G, X), C_{c}(G, X)\right\rangle_{C \times N} \\
& \subset \overline{\langle X \times N, X \times N\rangle_{C \times N}},
\end{aligned}
$$

which implies the desired containment since $p C_{c}(G, L(X)) q$ is dense in $p X_{N}^{G}(L(X)) q$.

\section{The Katayama Bimodule}

In this section we put no restrictions on our coaction (except that we continue to assume it satisfies the nondegeneracy condition of (2.1)!), and in this generality we introduce a right-Hilbert bimodule, which we call the Katayama bimodule, connecting the double dual coaction to the original; in the next section we restrict our attention to maximal coactions, and then the Katayama bimodule will be an imprimitivity bimodule.

Let $(A, G, \delta)$ be a coaction. Then as observed in [12, Corollary 2.6] there is always a canonical surjection

$$
\Phi_{A}=(\mathrm{id} \otimes \lambda) \circ \delta \times(1 \otimes M) \times(1 \otimes \rho): A \times{ }_{\delta} G \times{ }_{\widehat{\delta}} G \rightarrow A \otimes \mathcal{K}\left(L^{2}(G)\right),
$$

where $\lambda$ and $\rho$ are the left and right regular representations of $G$, and $M$ is the multiplication representation of $C_{0}(G)$, on $L^{2}(G)$. When there is no danger of confusion we will write $\Phi$ for $\Phi_{A}$. Also we will usually write $L^{2}$ for $L^{2}(G)$, and $\mathcal{K}$ for the compact operators on $L^{2}(G)$.

The external bimodule tensor product $A \otimes L^{2}$ is an $A \otimes \mathcal{K}-A$ imprimitivity bimodule, hence becomes a right-Hilbert $A \times_{\delta} G \times_{\widehat{\delta}} G-A$ bimodule when the left coefficient action is modified via $\Phi$. More precisely: 
Definition 4.1. For any coaction $(A, G, \delta)$, the Katayama bimodule $K(A)$ is the right-Hilbert $A \times{ }_{\delta} G \times \times_{\widehat{\delta}} G-A$ bimodule defined as the Hilbert $A$-module $A \otimes L^{2}$ with left $A \times{ }_{\delta} G \times{ }_{\widehat{\delta}} G$-module action given by

$$
f \cdot x=\Phi(f) x .
$$

We will now show that there is a $\widehat{\widehat{\delta}}-\delta$ compatible coaction $\delta_{K}$ on $K(A)$. First we recall some notation: $w_{G}$ is the unitary element of $M\left(C_{0}(G) \otimes C^{*}(G)\right)$ defined by the canonical embedding $G \hookrightarrow M\left(C^{*}(G)\right)$. For any $C^{*}$-algebras $C$ and $D$, $\Sigma: C \otimes D \rightarrow D \otimes C$ is the flip isomorphism. We let $\delta \otimes_{*}$ id $=($ id $\otimes \Sigma) \circ(\delta \otimes$ id $)$ denote the "external" tensor product coaction on either the bimodule $A \otimes L^{2}$ or the $C^{*}$-algebra $A \otimes \mathcal{K}$. Further let

$$
W=1 \otimes(M \otimes \mathrm{id})\left(w_{G}^{*}\right) \in M\left(A \otimes \mathcal{K} \otimes C^{*}(G)\right),
$$

where the first $M$ is the multiplication representation of $C_{0}(G)$.

Proposition 4.2. For any coaction $(A, G, \delta)$, the map

$$
\delta_{K}=W\left(\delta \otimes_{*} i d_{L^{2}}\right)
$$

is a $\widehat{\widehat{\delta}}-\delta$ compatible coaction on the Katayama bimodule $K(A)$.

Proof. The canonical surjection $\Phi$ is $\widehat{\widehat{\delta}}-\operatorname{Ad} W \circ\left(\delta \otimes_{*}\right.$ id $)$ equivariant, hence $\operatorname{Ad} W \circ$ $\left(\delta \otimes_{*}\right.$ id $)$ is a $\widehat{\hat{\delta}}-\operatorname{Ad} W \circ\left(\delta \otimes_{*}\right.$ id $)$ compatible coaction on the standard right-Hilbert bimodule ${ }_{A \times \delta} G \times{ }_{\hat{\delta}} G(A \otimes \mathcal{K})_{A \otimes \mathcal{K}}$. Moreover, $W$ is a $\left(\delta \otimes_{*}\right.$ id $)$-cocycle, so $W\left(\delta \otimes_{*}\right.$ id $)$ is an $\operatorname{Ad} W \circ\left(\delta \otimes_{*}\right.$ id $)-\left(\delta \otimes_{*}\right.$ id $)$ compatible coaction on $A \otimes \mathcal{K} A \otimes \mathcal{K}_{A \otimes \mathcal{K}}$. Also, $\delta \otimes_{*} \operatorname{id}_{L^{2}}$ is a $\left(\delta \otimes_{*} \operatorname{id}_{\mathcal{K}}\right)-\delta$ compatible coaction on the imprimitivity bimodule $A \otimes \mathcal{K}\left(A \otimes L^{2}\right)_{A}$. Combining these, we see that the balanced tensor product coaction

$$
\operatorname{Ad} W \circ\left(\delta \otimes_{*} \operatorname{id}_{\mathcal{K}}\right) \sharp_{A \otimes \mathcal{K}} W\left(\delta \otimes_{*} \operatorname{id}_{\mathcal{K}}\right) \sharp_{A \otimes \mathcal{K}}\left(\delta \otimes_{*} \operatorname{id}_{L^{2}}\right)
$$

on $\left(A \times_{\delta} G \times_{\widehat{\delta}} G\right) \otimes_{A \otimes \mathcal{K}}(A \otimes \mathcal{K}) \otimes_{A \otimes \mathcal{K}}\left(A \otimes L^{2}\right)$ is $\widehat{\widehat{\delta}}-\delta$ compatible.

To complete the proof, it suffices to show that the canonical right-Hilbert bimodule isomorphism

$$
(A \otimes \mathcal{K}) \otimes_{A \otimes \mathcal{K}}(A \otimes \mathcal{K}) \otimes_{A \otimes \mathcal{K}}\left(A \otimes L^{2}\right) \rightarrow K(A)
$$

takes the above triple tensor product coaction to the map $\delta_{K}$. In other words, we want to show that the outer rectangle of the diagram

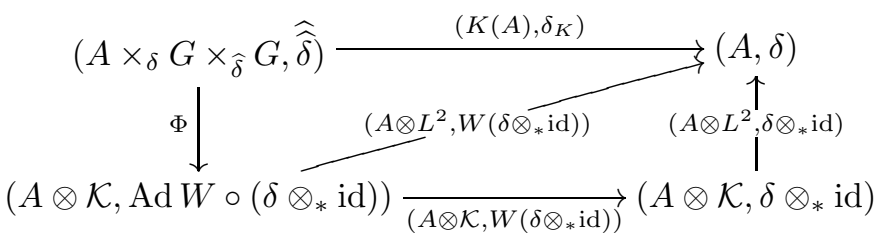

commutes. 
Let $\Psi:(A \otimes \mathcal{K}) \otimes A \otimes \mathcal{K}\left(A \otimes L^{2}\right) \rightarrow A \otimes L^{2}$ be the canonical isomorphism given by $\Psi(a \otimes k \otimes b \otimes \xi)=(a b \otimes k \xi)$ for $a, b \in A, k \in \mathcal{K}$, and $\xi \in L^{2}$. Then we have

$$
\begin{aligned}
& (\Psi \otimes \mathrm{id}) \circ\left(W\left(\delta \otimes_{*} \mathrm{id}\right) \sharp A \otimes \mathcal{K}\left(\delta \otimes_{*} \mathrm{id}\right)\right)((a \otimes k) \otimes(b \otimes \xi)) \\
& =(\Psi \otimes \mathrm{id}) \circ \Theta\left(W\left(\delta \otimes_{*} \mathrm{id}\right)(a \otimes k) \otimes\left(\delta \otimes_{*} \mathrm{id}\right)(b \otimes \xi)\right) \\
& =W\left(\delta \otimes_{*} \mathrm{id}\right)(a \otimes k)\left(\delta \otimes_{*} \mathrm{id}\right)(b \otimes \xi) \\
& =W\left(\delta \otimes_{*} \mathrm{id}\right)((a \otimes k)(b \otimes \xi)) \\
& =W\left(\delta \otimes_{*} \mathrm{id}\right) \circ \Psi((a \otimes k) \otimes(b \otimes \xi)),
\end{aligned}
$$

where the equality $(*)$ is easily verified on elementary tensors: for $a^{\prime}, b^{\prime} \in A, k^{\prime} \in \mathcal{K}$, $\xi^{\prime} \in L^{2}$, and $c, d \in C^{*}(G)$ we have

$$
\begin{aligned}
(\Psi \otimes \mathrm{id}) \circ \Theta\left(\left(a^{\prime} \otimes k^{\prime} \otimes c\right) \otimes\left(b^{\prime} \otimes \xi \otimes d\right)\right) & =(\Psi \otimes \mathrm{id})\left(\left(a^{\prime} \otimes k^{\prime}\right) \otimes\left(b^{\prime} \otimes \xi\right) \otimes c d\right) \\
& =a^{\prime} b^{\prime} \otimes k^{\prime} \xi \otimes c d \\
& =\left(a^{\prime} \otimes k^{\prime} \otimes c\right)\left(b^{\prime} \otimes \xi \otimes d\right) .
\end{aligned}
$$

This implies that the lower triangle commutes.

Commutativity of the upper triangle is much simpler: as in the proof of Theorem 3.1, it amounts to the fact that $W\left(\delta \otimes_{*}\right.$ id $)$ is a right-Hilbert bimodule homomorphism on $A \otimes L^{2}$ with left coefficient map $\operatorname{Ad} W \circ\left(\delta \otimes_{*}\right.$ id $)$.

We next show that the Katayama bimodule is a natural transformation from the functor " $\times G \times G$ " to the identity functor.

Theorem 4.3 (Naturality of Katayama). Let ${ }_{(A, \delta)}(X, \zeta)_{(B, \epsilon)}$ be a right-Hilbert bimodule coaction of a locally compact group $G$. Then the diagram

$$
\begin{aligned}
& \left(A \times{ }_{\delta} G \times \times_{\widehat{\delta}} G, \widehat{\widehat{\delta}}\right) \stackrel{\left(K(A), \delta_{K(A)}\right)}{\longrightarrow}(A, \delta) \\
& \left(X \times{ }_{\zeta} G \times{ }_{\widehat{\zeta}} G, \widehat{\widehat{\zeta}}\right) \downarrow \downarrow(X, \zeta) \\
& \left(B \times{ }_{\epsilon} G \times \times_{\widehat{\epsilon}} G, \widehat{\widehat{\epsilon}}\right) \underset{\left(K(B), \delta_{K(B)}\right)}{\longrightarrow}(B, \epsilon)
\end{aligned}
$$

commutes.

Proof. As in the proof of Theorem 3.1, we use the factorization strategy of [3] and [4]. So, first assume $(X, \zeta)$ is of the form $(B, \epsilon)$, where we have a nondegenerate $\delta-\epsilon$ equivariant homomorphism $\phi: A \rightarrow M(B)$. (Note that we cannot appeal to [4, Lemma 4.10] here because the Katayama bimodules are not imprimitivity bimodules.) The required diagram is the outer rectangle of the diagram

$$
\begin{aligned}
& \left(A \times_{\delta} G \times_{\widehat{\delta}} G, \widehat{\widehat{\delta}}\right) \stackrel{\left(K(A), \delta_{K(A)}\right)}{\longrightarrow}(A, \delta) \\
& \phi \times G \times G \downarrow \quad\left(B \otimes L^{2}, W(\epsilon \otimes * \text { id })\right) \downarrow \phi \\
& \left(B \times{ }_{\epsilon} G \times \times_{\widehat{\epsilon}} G, \widehat{\widehat{\epsilon}}\right) \underset{\left(K(B), \delta_{K(B)}\right)}{\longrightarrow}(B, \epsilon),
\end{aligned}
$$

where the bimodule on the diagonal is defined by the lower triangle; the coaction on the diagonal is appropriately compatible by equivariance of $\phi \times G \times G$. 
For the upper triangle, let $\Psi: K(A) \otimes_{A} B \rightarrow B \otimes L^{2}$ be the isomorphism given by $\Psi(a \otimes \xi \otimes b)=\phi(a) b \otimes \xi$. Then

$$
\begin{aligned}
(\Psi & \otimes \mathrm{id}) \circ\left(\delta_{K(A)} \sharp_{A} \epsilon\right)(a \otimes \xi \otimes b) \\
& =(\Psi \otimes \mathrm{id}) \circ \Theta\left(W\left(\delta \otimes_{*} \mathrm{id}\right)(a \otimes \xi) \otimes \epsilon(b)\right) \\
& =W(\mathrm{id} \otimes \Sigma)((\phi \otimes \mathrm{id})(\delta(a)) \epsilon(b) \otimes \xi) \\
& =W(\mathrm{id} \otimes \Sigma)(\epsilon(\phi(a)) \epsilon(b) \otimes \xi) \\
& =W(\mathrm{id} \otimes \Sigma)(\epsilon(\phi(a) b) \otimes \xi) \\
& =W\left(\epsilon \otimes_{*} \mathrm{id}\right)(\phi(a) b \otimes \xi) \\
& =W\left(\epsilon \otimes_{*} \mathrm{id}\right) \circ \Psi(a \otimes \xi \otimes b) .
\end{aligned}
$$

The equality $\left(^{*}\right)$ is verified by first computing, for $a^{\prime} \in A, \xi^{\prime} \in L^{2}, b^{\prime} \in B$, and $c, d \in C^{*}(G)$, that

$$
\begin{aligned}
(\Psi & \otimes \mathrm{id}) \circ \Theta\left(\left(a^{\prime} \otimes \xi^{\prime} \otimes c\right) \otimes\left(b^{\prime} \otimes d\right)\right) \\
& =(\Psi \otimes \mathrm{id})\left(a^{\prime} \otimes \xi^{\prime} \otimes b^{\prime} \otimes c d\right) \\
& =\phi\left(a^{\prime}\right) b^{\prime} \otimes \xi^{\prime} \otimes c d \\
& =\left(\phi\left(a^{\prime}\right) \otimes \xi^{\prime} \otimes c\right)\left(b^{\prime} \otimes 1 \otimes d\right) \\
& =(\phi \otimes \mathrm{id} \otimes \mathrm{id})\left(a^{\prime} \otimes \xi^{\prime} \otimes c\right)(\mathrm{id} \otimes \Sigma)\left(b^{\prime} \otimes d \otimes 1\right),
\end{aligned}
$$

and then using

$$
\begin{aligned}
(\phi \otimes \mathrm{id} \otimes \mathrm{id})\left(W\left(\delta \otimes_{*} \mathrm{id}\right)(a \otimes \xi)\right) & =W(\phi \otimes \mathrm{id} \otimes \mathrm{id})((\mathrm{id} \otimes \Sigma)(\delta(a) \otimes \xi)) \\
& =W(\mathrm{id} \otimes \Sigma)((\phi \otimes \mathrm{id})(\delta(a)) \otimes \xi)
\end{aligned}
$$

to see that

$$
\begin{aligned}
(\Psi & \otimes \mathrm{id}) \circ \Theta\left(W\left(\delta \otimes_{*} \mathrm{id}\right)(a \otimes \xi) \otimes \epsilon(b)\right) \\
& =(\phi \otimes \mathrm{id} \otimes \mathrm{id})\left(W\left(\delta \otimes_{*} \mathrm{id}\right)(a \otimes \xi)\right)(\mathrm{id} \otimes \Sigma)(\epsilon(b) \otimes 1) \\
& =W(\mathrm{id} \otimes \Sigma)((\phi \otimes \mathrm{id})(\delta(a)) \otimes \xi)(\mathrm{id} \otimes \Sigma)(\epsilon(b) \otimes 1) \\
& =W(\mathrm{id} \otimes \Sigma)(((\phi \otimes \mathrm{id})(\delta(a)) \otimes \xi)(\epsilon(b) \otimes 1)) \\
& =W(\mathrm{id} \otimes \Sigma)((\phi \otimes \mathrm{id})(\delta(a)) \epsilon(b) \otimes \xi) .
\end{aligned}
$$

Commutativity of the lower triangle amounts to the fact that $\delta_{K(B)}$ is a rightHilbert bimodule homomorphism with left coefficient map $\widehat{\widehat{\epsilon}}$.

Now assume that ${ }_{(A, \delta)}(X, \zeta)_{(B, \epsilon)}$ is a right-partial imprimitivity bimodule coaction. We can apply the linking algebra techniques of [4, Section 4.2] to conclude that the desired diagram commutes, provided we can show

$$
\langle p K(L(X)) q, p K(L(X)) q\rangle_{B} \subset \overline{\langle X, X\rangle_{B}} .
$$

But we have

$$
p K(L(X)) q=p\left(L(X) \otimes L^{2}\right) q=X \otimes L^{2},
$$

hence

$$
\langle p K(L(X)) q, p K(L(X)) q\rangle_{B}=\left\langle X \otimes L^{2}, X \otimes L^{2}\right\rangle_{B}=\langle X, X\rangle_{B},
$$

so we are done. 


\section{Full Mansfield imprimitivity}

In this section, we prove an equivariant version of Mansfield's Imprimitivity Theorem for full crossed products and maximal coactions. Our starting point is [5. Proposition 1.1], which provides the appropriate imprimitivity bimodule for dual coactions. Specifically, given an action $(B, G, \alpha)$ and a closed normal subgroup $N$ of $G$, there is a $B \times{ }_{\alpha} G \times \times_{\widehat{\alpha}} G \times \times_{\widehat{\alpha} \mid} N-B \times{ }_{\alpha} G \times \times_{\widehat{\alpha} \mid} G / N$ imprimitivity bimodule $Z_{G / N}^{G}\left(B \times{ }_{\alpha} G\right)$. This bimodule is a completion of $C_{c}(G \times G, B)$, with left action of $C_{c}(N \times G \times G, B)$ and right $C_{c}(G \times G / N, B)$-valued inner product given by

$$
\begin{aligned}
f \cdot z(s, t) & =\int_{N} \int_{G} f(n, r, t) \alpha_{r}\left(z\left(r^{-1} s, r^{-1} t n\right)\right) \Delta_{N}(n)^{1 / 2} d r d n, \\
\langle z, w\rangle_{B \times G \times G / N}(s, t N) & =\int_{N} \int_{G} \alpha_{r^{-1}}\left(z(r, r t n)^{*} w(r s, r t n)\right) d r d n .
\end{aligned}
$$

This left action is given on generators $b \in B, r \in G, h \in C_{c}(G) \subseteq C_{0}(G)$, and $n \in N$ by

$$
\begin{array}{ll}
(b \cdot z)(s, t)=b z(s, t) & (r \cdot z)(s, t)=\alpha_{r}\left(z\left(r^{-1} s, r^{-1} t\right)\right), \\
(h \cdot z)(s, t)=h(t) z(s, t) & (n \cdot z)(s, t)=z(s, t n) \Delta(n)^{1 / 2} .
\end{array}
$$

The next step is to define an $\operatorname{Inf} \widehat{\widehat{\alpha}} \mid-\widehat{\alpha}^{\text {dec }}$ compatible coaction $\delta_{Z}$ on $Z_{G / N}^{G}\left(B \times{ }_{\alpha} G\right)$. A related construction appears in [4. Lemma 5.7], where it was deduced from [7], and the context there is reduced crossed products. Thus we feel it is best to construct the bimodule coaction from scratch here, and this will take a little bit of work.

In the following lemma, we identify $C_{c}(G \times G, B) \odot C^{*}(G)$ with an inductivelimit-dense subspace of $C_{c}\left(G \times G, B \otimes C^{*}(G)\right)$, and hence with a dense subspace of the bimodule $Z_{G / N}^{G}\left(\left(B \otimes C^{*}(G)\right) \times_{\alpha \otimes \text { id }} G\right)$, by identifying an elementary tensor $z \otimes c$ with the function defined by

$$
(z \otimes c)(s, t)=z(s, t) \otimes c .
$$

Lemma 5.1. The identity map on $C_{c}(G \times G, B) \odot C^{*}(G)$ extends to an imprimitivity bimodule surjection

$$
\begin{aligned}
\Gamma:\left(B \otimes C^{*}(G)\right) \times G \times G \times N & \left(Z_{G / N}^{G}\left(\left(B \otimes C^{*}(G)\right) \times{ }_{\alpha \otimes i d} G\right)\right)_{\left(B \otimes C^{*}(G)\right) \times G \times G / N} \\
& \rightarrow(B \times G \times G \times N) \otimes C^{*}(G) \\
& \left(Z_{G / N}^{G}\left(B \times{ }_{\alpha} G\right) \otimes C^{*}(G)\right)_{(B \times G \times G / N) \otimes C^{*}(G)} .
\end{aligned}
$$

Proof. The right-hand coefficient homomorphism

$$
\psi:\left(B \otimes C^{*}(G)\right) \times_{\alpha \otimes \mathrm{id}} G \times \widehat{\alpha \otimes \mathrm{id} \mid} G / N \rightarrow\left(B \times_{\alpha} G \times_{\widehat{\alpha} \mid} G / N\right) \otimes C^{*}(G)
$$

is the integrated form $\psi=\left(k_{B} \otimes \mathrm{id}\right) \times\left(k_{G} \otimes 1\right) \times\left(k_{C(G / N)} \otimes 1\right)$, where $\left(k_{B}, k_{G}, k_{C(G / N)}\right)$ are the canonical covariant maps of $\left(B, G, C_{0}(G / N)\right)$ into $M\left(B \times{ }_{\alpha} G \times \times_{\alpha} G / N\right)$. It is clear that $\psi$ maps generators to generators, so is a surjection.

Similarly, the left-hand coefficient homomorphism

$$
\phi:\left(B \otimes C^{*}(G)\right) \times_{\alpha \otimes \mathrm{id}} G \times \underset{\alpha \otimes \mathrm{id}}{\widehat{\alpha}} G \times \underset{\alpha \otimes \mathrm{id} \mid}{\widehat{N}} N \rightarrow\left(B \times_{\alpha} G \times_{\widehat{\alpha}} G \times \times_{\widehat{\hat{\alpha}} \mid} N\right) \otimes C^{*}(G)
$$

is the surjective integrated form $\phi=\left(\ell_{B} \otimes \mathrm{id}\right) \times\left(\ell_{G} \otimes 1\right) \times\left(\ell_{C(G)} \otimes 1\right) \times\left(\ell_{N} \otimes 1\right)$, where $\left(\ell_{B}, \ell_{G}, \ell_{C(G)}, \ell_{N}\right)$ are the canonical covariant maps of $\left(B, G, C_{0}(G), N\right)$ into $M\left(B \times{ }_{\alpha} G \times \times_{\widehat{\alpha}} G \times{ }_{\widehat{\hat{\alpha}} \mid} N\right)$. 
For the bimodule map itself, a routine computation on elements of $C_{c}(G \times G, B) \odot$ $C^{*}(G)$ shows that

$$
\langle z \otimes c, w \otimes d\rangle_{\left(B \otimes C^{*}(G)\right) \times G \times G / N}(s, t N)=\langle z, w\rangle_{B \times G \times G / N}(s, t N) \otimes c^{*} d,
$$

so we have

$$
\psi\left(\langle z \otimes c, w \otimes d\rangle_{\left(B \otimes C^{*}(G)\right) \times G \times G / N}\right)=\langle z, w\rangle_{B \times G \times G / N} \otimes c^{*} d .
$$

Therefore the identity map on $C_{c}(G \times G, B) \odot C^{*}(G)$ extends to a bounded linear surjection $\Gamma: Z_{G / N}^{G}\left(\left(B \otimes C^{*}(G)\right) \times_{\alpha \otimes \text { id }} G\right) \rightarrow Z_{G / N}^{G}\left(B \times_{\alpha} G\right) \otimes C^{*}(G)$.

Another routine calculation (using (5.2) ) shows that for any generator $x$ from $B \otimes C^{*}(G), G, C_{0}(G)$, or $N$, the left module actions on elementary tensors satisfy

$$
\Gamma(x \cdot(z \otimes c))=\phi(x) \cdot \Gamma(z \otimes c) .
$$

Thus $\Gamma$ is a right-Hilbert bimodule homomorphism with coefficient maps $\phi$ and $\psi$.

In particular, Lemma 5.1 gives an embedding of $C_{c}\left(G \times G, B \otimes C^{*}(G)\right)$ in $Z_{G / N}^{G}\left(B \times{ }_{\alpha} G\right) \otimes C^{*}(G)$; by standard function space techniques (see for example [4 Appendix C]), we also get an embedding of $C_{c}\left(G \times G, M^{\beta}\left(B \otimes C^{*}(G)\right)\right)$ in $M\left(Z_{G / N}^{G}\left(B \times{ }_{\alpha} G\right) \otimes C^{*}(G)\right)$. Thus (as in the proof of the next proposition), we can use the bimodule operations from $Z_{G / N}^{G}\left(\left(B \otimes C^{*}(G)\right) \times_{\alpha \otimes \text { id }} G\right)$ in calculations with $C_{c^{-}}$-functions, although we regard the functions as elements of $Z_{G / N}^{G}\left(B \times{ }_{\alpha} G\right) \otimes$ $C^{*}(G)$.

Proposition 5.2. Let $(B, G, \alpha)$ be an action, and let $N$ be a closed normal subgroup of $G$. The map $\delta_{Z}: C_{c}(G \times G, B) \rightarrow C_{c}\left(G \times G, M^{\beta}\left(B \otimes C^{*}(G)\right)\right)$ defined by

$$
\delta_{Z}(z)(s, t)=z(s, t) \otimes t^{-1} s
$$

extends uniquely to an $\operatorname{Inf} \widehat{\widehat{\hat{\alpha}}}-\widehat{\alpha}^{\text {dec }}$ compatible coaction on $Z_{G / N}^{G}\left(B \times{ }_{\alpha} G\right)$.

Proof. Since $Z_{G / N}^{G}\left(B \times{ }_{\alpha} G\right)$ is an imprimitivity bimodule, and since the coefficient maps are coactions, it suffices to show that $\delta_{Z}$ satisfies the coaction identity and preserves the left module actions and the right inner products. For the coaction identity, first note that for $y \otimes c \in C_{c}(G \times G, B) \odot C^{*}(G)$, we have

$$
\begin{aligned}
\left(\delta_{Z} \otimes \mathrm{id}\right)(y \otimes c) & \in C_{c}\left(G \times G, M^{\beta}\left(B \otimes C^{*}(G)\right) \odot C^{*}(G)\right) \\
& \subset C_{c}\left(G \times G, M^{\beta}\left(B \otimes C^{*}(G) \otimes C^{*}(G)\right)\right),
\end{aligned}
$$

with

$$
\begin{aligned}
\left(\delta_{Z} \otimes \mathrm{id}\right)(y \otimes c)(s, t) & =\left(\delta_{Z}(y) \otimes c\right)(s, t) \\
& =\delta_{Z}(y)(s, t) \otimes c \\
& =y(s, t) \otimes t^{-1} s \otimes c \\
& =(\mathrm{id} \otimes \Sigma)\left(y(s, t) \otimes c \otimes t^{-1} s\right) \\
& =(\mathrm{id} \otimes \Sigma)\left((y \otimes c)(s, t) \otimes t^{-1} s\right) .
\end{aligned}
$$

It follows from density and continuity for the inductive-limit topologies that for $z \in C_{c}(G \times G, B)$, we have $\left(\delta_{Z} \otimes \mathrm{id}\right) \circ \delta_{Z}(z) \in C_{c}\left(G \times G, M^{\beta}\left(B \otimes C^{*}(G) \otimes C^{*}(G)\right)\right)$, 
with

$$
\begin{aligned}
\left(\delta_{Z} \otimes \mathrm{id}\right) \circ \delta_{Z}(z)(s, t) & =(\mathrm{id} \otimes \Sigma)\left(\delta_{Z}(z)(s, t) \otimes t^{-1} s\right) \\
& =(\mathrm{id} \otimes \Sigma)\left(z(s, t) \otimes t^{-1} s \otimes t^{-1} s\right) \\
& =z(s, t) \otimes t^{-1} s \otimes t^{-1} s \\
& =\left(\mathrm{id} \otimes \delta_{G}\right)\left(z(s, t) \otimes t^{-1} s\right) \\
& =\left(\mathrm{id} \otimes \delta_{G}\right)\left(\delta_{Z}(z)(s, t)\right) \\
& =\left(\mathrm{id} \otimes \delta_{G}\right) \circ \delta_{Z}(z)(s, t) .
\end{aligned}
$$

For the left module actions, if $f \in C_{c}(N \times G \times G, B) \subset B \times{ }_{a} G \times \times_{\widehat{\alpha}} G \times \widehat{\widehat{\alpha} \mid} N$ and $z \in C_{c}(G \times G, B)$ we have

$$
\begin{aligned}
& \left(\operatorname{Inf} \widehat{\widehat{\hat{\alpha}}}(f) \cdot \delta_{Z}(z)\right)(s, t) \\
& \quad=\int_{N} \int_{G} \operatorname{Inf} \widehat{\widehat{\hat{\alpha}}}(f)(n, r, t)(\alpha \otimes \mathrm{id})_{r}\left(\delta_{Z}(z)\left(r^{-1} s, r^{-1} t n\right)\right) \Delta(n)^{1 / 2} d r d n \\
& =\int_{N} \int_{G}(f(n, r, t) \otimes n)\left(\alpha_{r} \otimes \mathrm{id}\right)\left(z\left(r^{-1} s, r^{-1} t n\right) \otimes n^{-1} t^{-1} s\right) \Delta(n)^{1 / 2} d r d n \\
& =\int_{N} \int_{G}(f(n, r, t) \otimes n)\left(\alpha_{r}\left(z\left(r^{-1} s, r^{-1} t n\right)\right) \otimes n^{-1} t^{-1} s\right) \Delta(n)^{1 / 2} d r d n \\
& =\int_{N} \int_{G}\left(f(n, r, t) \alpha_{r}\left(z\left(r^{-1} s, r^{-1} t n\right)\right) \otimes t^{-1} s\right) \Delta(n)^{1 / 2} d r d n \\
& =\int_{N} \int_{G} f(n, r, t) \alpha_{r}\left(z\left(r^{-1} s, r^{-1} t n\right)\right) \Delta(n)^{1 / 2} d r d n \otimes t^{-1} s \\
& =(f \cdot z)(s, t) \otimes t^{-1} s=\delta_{Z}(f \cdot z)(s, t) .
\end{aligned}
$$

Finally, for the inner products, if $z, w \in C_{c}(G \times G, B)$, then

$$
\begin{aligned}
\left\langle\delta_{Z}(z), \delta_{Z}(w)\right\rangle_{M\left((B \times G \times G / N) \otimes C^{*}(G)\right)}(s, t N) & \\
& =\int_{N} \int_{G}(\alpha \otimes \mathrm{id})_{r^{-1}}\left(\delta_{Z}(z)(r, r t n)^{*} \delta_{Z}(w)(r s, r t n)\right) d r d n \\
& =\int_{N} \int_{G}\left(\alpha_{r^{-1}} \otimes \mathrm{id}\right)\left(\left(z(r, r t n) \otimes n^{-1} t^{-1}\right)^{*}\left(w(r s, r t n) \otimes n^{-1} t^{-1} s\right)\right) d r d n \\
& =\int_{N} \int_{G}\left(\alpha_{r^{-1}} \otimes \mathrm{id}\right)\left(z(r, r t n)^{*} w(r s, r t n) \otimes s\right) d r d n \\
& =\int_{N} \int_{G} \alpha_{r^{-1}}\left(z(r, r t n)^{*} w(r s, r t n)\right) d r d n \otimes s \\
& =\langle z, w\rangle_{B \times G \times G / N}(s, t N) \otimes s=\widehat{\alpha}^{\operatorname{dec}}\left(\langle z, w\rangle_{B \times G \times G / N}\right)(s, t N) .
\end{aligned}
$$

Theorem 5.3 (Full Mansfield imprimitivity). For any maximal coaction $(A, G, \delta)$ and any closed normal subgroup $N$ of $G$, there exists an

$$
A \times{ }_{\delta} G \times \times_{\widehat{\delta} \mid} N-A \times{ }_{\delta \mid} G / N
$$

imprimitivity bimodule $Y_{G / N}^{G}(A)$ and an $\operatorname{Inf} \widehat{\widehat{\delta}} \mid-\delta^{\text {dec }}$ compatible coaction $\delta_{Y}$ of $G$ on $Y_{G / N}^{G}(A)$.

We refer to $Y_{G / N}^{G}(A)$ as the Mansfield bimodule. 
Proof. Let $(C, \epsilon)=\left(A \times{ }_{\delta} G \times \widehat{\delta} G, \widehat{\widehat{\delta}}\right)$. Then since $\delta$ is maximal, $K(A)$ is an equivariant $C-A$ imprimitivity bimodule, so the following diagram serves to define $Y_{G / N}^{G}(A)$ and $\delta_{Y}$ :

$$
\begin{aligned}
& \left(A \times_{\delta} G \times \times_{\widehat{\delta} \mid} N, \operatorname{Inf} \widehat{\widehat{\delta}} \mid\right) \stackrel{\left(Y_{G / N}^{G}(A), \delta_{Y}\right)}{\longrightarrow}\left(A \times_{\delta \mid} G / N, \delta^{\mathrm{dec}}\right)
\end{aligned}
$$

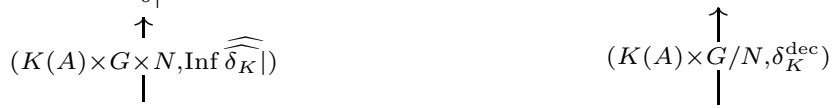

$$
\begin{aligned}
& \left(C \times{ }_{\epsilon} G \times \times_{\widehat{\epsilon} \mid} N, \operatorname{Inf} \widehat{\widehat{\epsilon}}\right) \underset{\left(Z_{G / N}^{G}(C), \delta_{Z}\right)}{\longrightarrow}\left(C \times \times_{\epsilon \mid} G / N, \epsilon^{\mathrm{dec}}\right) .
\end{aligned}
$$

\section{Naturality of the Mansfield Bimodule}

In this section we show that the Mansfield bimodule is a natural equivalence between the functors " $\times G \times N$ " and " $\times G / N$ ", when those functors are restricted to $\mathcal{C}_{m}(G)$. The first step is to resolve two potential ambiguities among our bimodules.

Proposition 6.1. For any dual coaction $(A, G, \delta)$, we have

$$
\left(K(A), \delta_{K}\right) \cong\left(Z_{G / G}^{G}(A), \delta_{Z}\right) .
$$

Proof. Let $(B, G, \alpha)$ be an action such that $\left(B \times{ }_{\alpha} G, \widehat{\alpha}\right)=(A, \delta)$, and define a linear map $\Psi: C_{c}(G, B) \odot C_{c}(G) \rightarrow C_{c}(G \times G, B)$ by

$$
\Psi(f \otimes \xi)(s, t)=f(s) \xi(t) .
$$

To see that $\Psi$ extends to an imprimitivity bimodule isomorphism of $K(A)$ onto $Z_{G / G}^{G}(A)$, it suffices to show that it preserves the right inner products and the left module actions of the generators of $B \times{ }_{\alpha} G \times \times_{\widehat{\alpha}} G \times{ }_{\widehat{\alpha}} G$. For the inner products, we have

$$
\begin{aligned}
\langle\Psi & (f \otimes \xi), \Psi(g \otimes \eta)\rangle_{B \times G}(s) \\
& =\iint \alpha_{t^{-1}}\left(\Psi(f \otimes \xi)(t, r)^{*} \Psi(g \otimes \eta)(t s, r)\right) d r d t \\
& =\iint \alpha_{t^{-1}}\left((f(t) \xi(r))^{*} g(t s) \eta(r)\right) d r d t \\
& =\iint \alpha_{t^{-1}}\left(f(t)^{*} \overline{\xi(r)} g(t s) \eta(r)\right) d r d t \\
& =\iint \alpha_{t^{-1}}\left(f(t)^{*} g(t s)\right) \overline{\xi(r)} \eta(r) d r d t \\
& =\int \alpha_{t^{-1}}\left(f(t)^{*} g(t s)\right) d t \int \overline{\xi(r)} \eta(r) d r \\
& =\left(f^{*} g\right)(s)\langle\xi, \eta\rangle=\langle f, g\rangle_{B \times G}(s)\langle\xi, \eta\rangle \\
& =\left(\langle f, g\rangle_{B \times G}\langle\xi, \eta\rangle\right)(s)=\langle f \otimes \xi, g \otimes \eta\rangle_{B \times G}(s) .
\end{aligned}
$$

Recall that the left action on $K(A)$ is implemented by the canonical surjection $\Phi=(\mathrm{id} \otimes \lambda) \circ \widehat{\alpha} \times(1 \otimes M) \times(1 \otimes \rho)$; the left action of the generators on $Z_{G / G}^{G}(A)$ 
is given by (5.2). Thus, if $\left(i_{B}, i_{G}\right)$ denotes the canonical covariant homomorphism of $(B, G)$ into $M\left(B \times{ }_{\alpha} G\right)$, for $b \in B$ we have

$$
\begin{aligned}
\Psi & (b \cdot(f \otimes \xi))(s, t) \\
& =\Psi\left((\operatorname{id} \otimes \lambda) \circ \widehat{\alpha}\left(i_{B}(b)\right)(f \otimes \xi)\right)(s, t) \\
& =\Psi\left(\left(i_{B}(b) \otimes 1\right)(f \otimes \xi)\right)(s, t) \\
& =\Psi\left(i_{B}(b) f \otimes \xi\right)(s, t)=\left(i_{B}(b) f\right)(s) \xi(t) \\
& =b f(s) \xi(t)=b \Psi(f \otimes \xi)(s, t) \\
& =(b \cdot \Psi(f \otimes \xi))(s, t),
\end{aligned}
$$

and for $r \in G$ we have

$$
\begin{aligned}
\Psi(r & \cdot(f \otimes \xi))(s, t) \\
& =\Psi\left((\mathrm{id} \otimes \lambda) \circ \widehat{\alpha}\left(i_{G}(r)\right)(f \otimes \xi)\right)(s, t) \\
& =\Psi\left(\left(i_{G}(r) \otimes \lambda_{r}\right)(f \otimes \xi)\right)(s, t) \\
& =\Psi\left(i_{G}(r) f \otimes \lambda_{r} \xi\right)(s, t)=\left(i_{G}(r) f\right)(s)\left(\lambda_{r} \xi\right)(t) \\
& =\alpha_{r}\left(f\left(r^{-1} s\right)\right) \xi\left(r^{-1} t\right)=\alpha_{r}\left(\Psi(f \otimes \xi)\left(r^{-1} s, r^{-1} t\right)\right) \\
& =(r \cdot \Psi(f \otimes \xi))(s, t) .
\end{aligned}
$$

Similarly, for $h \in C_{c}(G) \subset C_{0}(G)$ we have

$$
\begin{aligned}
\Psi(h \cdot(f \otimes \xi))(s, t) & =\Psi\left(\left(1 \otimes M_{h}\right)(f \otimes \xi)\right)(s, t) \\
& =\Psi\left(f \otimes M_{h} \xi\right)(s, t) \\
& =f(s)\left(M_{h} \xi\right)(t)=f(s) h(t) \xi(t) \\
& =h(t) f(s) \xi(t)=h(t) \Psi(f \otimes \xi)(s, t) \\
& =(h \cdot \Psi(f \otimes \xi))(s, t),
\end{aligned}
$$

and for $u \in G$ (meaning the "outermost" $G$ in $B \times{ }_{\alpha} G \times \times_{\widehat{\alpha}} G \times{ }_{\widehat{\alpha}} G$ ) we have

$$
\begin{aligned}
\Psi(u \cdot(f \otimes \xi))(s, t) & =\Psi\left(\left(1 \otimes \rho_{u}\right)(f \otimes \xi)\right)(s, t) \\
& =\Psi\left(f \otimes \rho_{u} \xi\right)(s, t)=f(s)\left(\rho_{u} \xi\right)(t) \\
& =f(s) \xi(t u) \Delta(u)^{1 / 2}=\Psi(f \otimes \xi)(s, t u) \Delta(u)^{1 / 2} \\
& =(u \cdot \Psi(f \otimes \xi))(s, t)
\end{aligned}
$$


Finally, we show $\Psi$ is $\delta_{K}-\delta_{Z}$ equivariant. Let $f \in C_{c}(G, B) \subset B \times{ }_{\alpha} G$ and $\xi \in C_{c}(G) \subset L^{2}$. Then

$$
\begin{aligned}
(\Psi \otimes \mathrm{id}) \circ \delta_{K}(f \otimes \xi)(s, t) & =(\Psi \otimes \mathrm{id})(W(\widehat{\alpha} \otimes * \mathrm{id})(f \otimes \xi))(s, t) \\
& =(\Psi \otimes \mathrm{id})\left(w_{G}^{*} \cdot(\mathrm{id} \otimes \Sigma)(\widehat{\alpha}(f) \otimes \xi)\right)(s, t) \\
& =\left(w_{G}^{*} \cdot(\Psi \otimes \mathrm{id}) \circ(\mathrm{id} \otimes \Sigma)(\widehat{\alpha}(f) \otimes \xi)\right)(s, t) \\
& =\left(1 \otimes w_{G}^{*}(t)\right)((\Psi \otimes \mathrm{id}) \circ(\mathrm{id} \otimes \Sigma)(\widehat{\alpha}(f) \otimes \xi))(s, t) \\
& =\left(1 \otimes t^{-1}\right) \widehat{\alpha}(f)(s) \xi(t) \\
& =\left(1 \otimes t^{-1}\right)(f(s) \otimes s) \xi(t) \\
& =f(s) \xi(t) \otimes t^{-1} s \\
& =\Psi(f \otimes \xi)(s, t) \otimes t^{-1} s \\
& =\delta_{Z} \circ \Psi(f \otimes \xi)(s, t),
\end{aligned}
$$

where the equality $\left(^{*}\right)$ is verified by temporarily replacing $\widehat{\alpha}(f)$ by an elementary tensor $g \otimes c \in C_{c}(G, B) \odot C^{*}(G)$, computing that

$$
\begin{aligned}
(\Psi \otimes \text { id }) \circ(\text { id } \otimes \Sigma)(g \otimes c \otimes \xi)(s, t) & =(\Psi \otimes \text { id })(g \otimes \xi \otimes c)(s, t) \\
& =(\Psi(g \otimes \xi) \otimes c)(s, t) \\
& =\Psi(g \otimes \xi)(s, t) \otimes c \\
& =g(s) \xi(t) \otimes c \\
& =(g(s) \otimes c) \xi(t) \\
& =(g \otimes c)(s) \xi(t),
\end{aligned}
$$

and then appealing to continuity and density for the inductive-limit topologies.

Corollary 6.2. For any maximal coaction $(A, G, \delta)$, we have

$$
\left(Y_{G / G}^{G}(A), \delta_{Y}\right) \cong\left(K(A), \delta_{K}\right) .
$$

Proof. Applying naturality of Katayama (Theorem 4.3) to the Katayama bimodule itself gives

$$
K(A) \times_{\delta_{K}} G \times \times_{\widehat{\delta_{K}}} G \otimes_{A \times G \times G} K(A) \cong K\left(A \times_{\delta} G \times_{\widehat{\delta}} G\right) \otimes_{A \times G \times G} K(A),
$$

equivariantly for the appropriate coactions. Since $\delta$ is maximal, $K(A)$ is an imprimitivity bimodule, so it follows that $K(A) \times{ }_{\delta_{K}} G \times \widehat{\delta_{K}} G$ is equivariantly isomorphic to $K\left(A \times{ }_{\delta} G \times \times_{\widehat{\delta}} G\right)$. Combining this with Proposition 6.1 (applied to $\widehat{\widehat{\delta}}$ ) gives

$$
K(A) \times_{\delta_{K}} G \times \times_{\widehat{\delta_{K}}} G \cong Z_{G / G}^{G}\left(A \times{ }_{\delta} G \times \times_{\widehat{\delta}} G\right),
$$

equivariantly for the appropriate coactions; the proposition now follows immediately from this and the definition of $Y_{G / G}^{G}(A)$ (Diagram (5.3)).

We now establish a curious duality between Mansfield and Green imprimitivity. 
Proposition 6.3 (Factorization of $\left.Z_{G / N}^{G}\right)$. For any dual coaction $(A, G, \delta)$ and any closed normal subgroup $N$ of $G$, the diagram

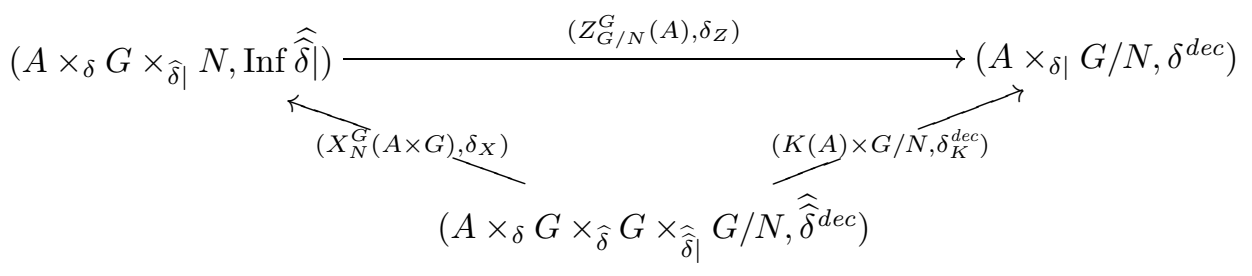

commutes.

Proof. Let $(B, G, \alpha)$ be an action such that $\left(B \times_{\alpha} G, \widehat{\alpha}\right)=(A, \delta)$. Then the desired diagram is the upper left triangle of the diagram

$$
\begin{aligned}
& \left(A \times_{\delta} G \times \times_{\widehat{\delta}} G \times \times_{\widehat{\delta} \mid} G / N, \widehat{\widehat{\delta}}^{\mathrm{dec}}\right) \stackrel{\left(X_{N}^{G}(A \times G), \delta_{X}\right)}{\longrightarrow}\left(B \times{ }_{\alpha} G \times \times_{\widehat{\alpha}} G \times \times_{\widehat{\hat{\alpha}}}|N, \operatorname{Inf} \widehat{\widehat{\hat{\alpha}}}|\right)
\end{aligned}
$$

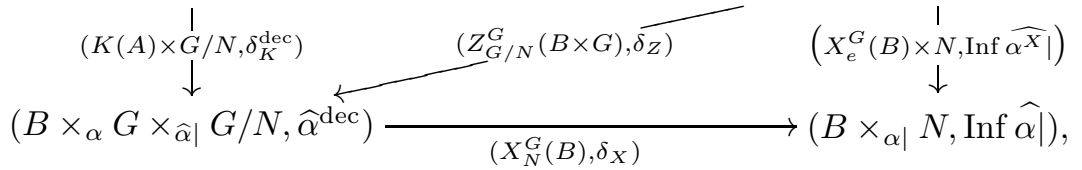

where $\alpha^{X}$ is the $\widehat{\widehat{\alpha}}-\alpha$ compatible action on $X_{e}^{G}(B)$ from [1, Theorem 1]. Without the equivariance, the lower right triangle commutes by [5. Theorem 3.1]. Equivariance is proven in [4, Theorem 5.1], albeit in the context of reduced crossed products; the argument given there carries over with no significant change, since it only uses $C_{c}$-functions and pre-right-Hilbert bimodules.

Next, note that the lower triangle in the special case $N=G$ gives us

$$
\left(Z_{G / G}^{G}\left(B \times{ }_{\alpha} G\right), \delta_{X}\right) \cong\left(X_{e}^{G}(B) \times_{\alpha^{X}} G, \widehat{\alpha^{X}}\right),
$$

so by equivariance and Proposition 6.1 we get

$$
\left(K(A) \times_{\delta_{K} \mid} G / N, \delta_{K}^{\mathrm{dec}}\right) \cong\left(X_{e}^{G}(B) \times_{\alpha^{x}} G \times{\widehat{\alpha^{X}} \mid} G / N,{\widehat{\alpha^{X}}}^{\mathrm{dec}}\right) .
$$

Using this to replace the left-hand vertical arrow in the diagram above reduces the outer rectangle to naturality of Green (Theorem 3.1), applied to the $A \times{ }_{\delta} G-B$ imprimitivity bimodule $X_{e}^{G}(B)$; this finishes the proof.

Corollary 6.4 (Factorization of $\left.Y_{G / N}^{G}\right)$. For any maximal coaction $(A, G, \delta)$ and any closed normal subgroup $N$ of $G$, the diagram

$$
\begin{aligned}
& \left(A \times_{\delta} G \times \times_{\widehat{\delta} \mid} N, \operatorname{Inf} \widehat{\widehat{\delta}} \mid\right) \longrightarrow\left(A \times_{\delta \mid} G / N, \delta^{\text {dec }}\right) \\
& \overbrace{\left(X_{N}^{G}\right.}(A \times G), \delta_{X}) \quad\left(K(A) \times G / \widehat{\left.N, \delta_{K}^{\text {dec }}\right)}\right. \\
& \left(A \times_{\delta} G \times \times_{\widehat{\delta}} G \times \widehat{\widehat{\delta}}_{\widehat{\delta} \mid} G / N, \widehat{\widehat{\delta}^{d e c}}\right)
\end{aligned}
$$

commutes. 
Proof. To simplify the notation, let $(C, G, \epsilon)=\left(A \times_{\delta} G \times_{\widehat{\delta}} G, G, \widehat{\widehat{\delta}}\right)$. Then the desired diagram is the top triangle of the diagram

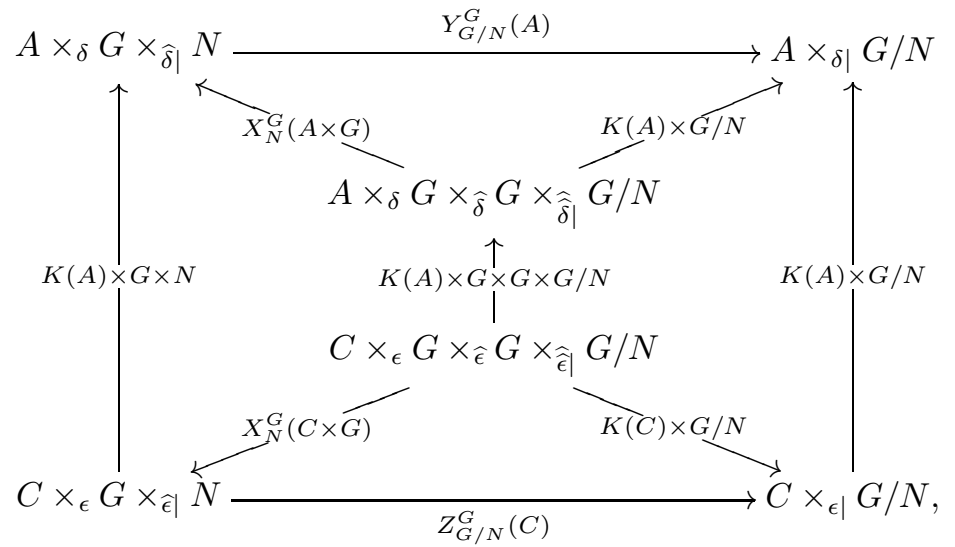

which is to be interpreted as an equivariant diagram, although we have omitted the appropriate coactions for clarity. The bottom triangle commutes by Proposition 6.3 The outer rectangle commutes by definition of $Y_{G / N}^{G}(A)$ (Diagram (5.3)). The left quadrilateral commutes by naturality of Green (Theorem [3.1), applied to the $C \times{ }_{\epsilon} G-A \times{ }_{\delta} G$ imprimitivity bimodule $K(A) \times{ }_{\delta_{K}} G$. Finally, the right quadrilateral commutes by naturality of Katayama (Theorem 4.3), applied to $K(A)$ itself, together with functoriality of " $\times G / N$ ".

We can use the above result to clear up another apparent ambiguity, which arises for dual coactions.

Proposition 6.5. For any dual coaction $(A, G, \delta)$ and any closed normal subgroup $N$ of $G$, we have

$$
\left(Y_{G / N}^{G}(A), \delta_{Y}\right) \cong\left(Z_{G / N}^{G}(A), \delta_{Z}\right) .
$$

Proof. This is immediate from Proposition 6.3 and Corollary 6.4 since both bimodules factor as

$$
\widetilde{X_{N}^{G}}(A \times G) \otimes_{A \times{ }_{\delta} G \times{ }_{\hat{\delta}}} G \times G / N(K(A) \times G / N),
$$

equivariantly for the appropriate coactions.

We are now ready to prove that the Mansfield bimodule is natural. We have factored Mansfield as a composition of Green and Katayama, each of which is natural, and this is what makes it work.

Theorem 6.6 (Naturality of Mansfield). Let ${ }_{(A, \delta)}(X, \zeta)_{(B, \epsilon)}$ be a right-Hilbert bimodule coaction of a locally compact group $G$, and let $N$ be a closed normal subgroup of $G$. If $\delta$ and $\epsilon$ are maximal, then the following diagram commutes:

$$
\begin{aligned}
& \left(A \times{ }_{\delta} G \times{ }_{\widehat{\delta} \mid} N, \operatorname{Inf} \widehat{\widehat{\delta}} \mid\right) \stackrel{\left(Y_{G / N}^{G}(A), \delta_{Y}\right)}{\longrightarrow}\left(A \times_{\delta \mid} G / N, \delta^{\text {dec }}\right) \\
& (X \times G \times N, \operatorname{Inf} \widehat{\widehat{\zeta} \mid})\rfloor\rfloor\left(X \times G / N, \zeta^{d e c}\right) \\
& \left(B \times_{\epsilon} G \times \times_{\widehat{\epsilon} \mid} N, \operatorname{Inf} \widehat{\widehat{\epsilon}}\right) \underset{\left(Y_{G / N}^{G}(B), \delta_{Y}\right)}{\longrightarrow}\left(B \times \times_{\epsilon \mid} G / N, \epsilon^{d e c}\right)
\end{aligned}
$$


Proof. The desired diagram is the outer rectangle of the diagram

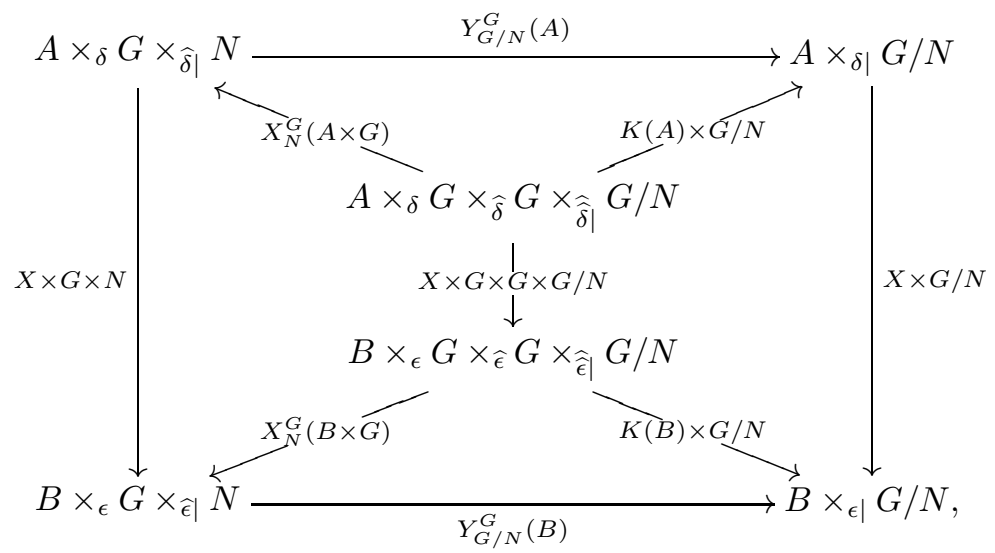

which is again to be interpreted equivariantly for the appropriate coactions. The top and bottom triangles commute by factorization of $Y_{G / N}^{G}$ (Corollary [6.4), the left quadrilateral commutes by naturality of Green (Theorem 3.1), and the right quadrilateral commutes by naturality of Katayama (Theorem 4.3) and functoriality of " $\times G / N$ ".

\section{Preservation of maximality}

In this section, partly as an application of the results of the preceding two, we show that the functors involved in the Full Mansfield Imprimitivity Theorem (Theorem 5.3) can be regarded as having the category $\mathcal{C}_{m}(G)$ of maximal coactions of $G$ not only for their domain, but also their codomain. This involves verifying that maximality of coactions is preserved under various operations; in doing so we freely use the facts that dual coactions are maximal, and that maximality is preserved by Morita-Rieffel equivalence ([2 Propositions 3.4 and 3.5]). We end the section with another preservation-of-maximality result which is not needed for the purpose of investigating the codomains of our functors, but which is of obvious interest in this circle of ideas.

First recall that if $(A, G, \alpha, N, \tau)$ is a twisted action in the sense of Green [8], then there is a natural "dual coaction" $\widetilde{\alpha}$ of $G / N$ on the twisted crossed product $A \times{ }_{\alpha, N} G$.

Proposition 7.1. Let $(A, G, \alpha, N, \tau)$ be a twisted action. Then the dual coaction $\left(A \times_{\alpha, N} G, G / N, \widetilde{\alpha}\right)$ is maximal.

Proof. By Echterhoff's version of the Stabilization Trick [1 Theorem 1], the twisted action of $(G, N)$ is Morita-Rieffel equivalent to an inflated action $(B, G, \operatorname{Inf} \beta)$, where $\beta$ is an action of $G / N$ on $B$ (and this inflated action is trivially twisted over $N$ ). Thus the dual coactions $\widehat{\alpha}$ and $\widehat{\operatorname{Inf} \beta}$ are Morita-Rieffel equivalent, hence so are the corresponding coactions $\widetilde{\alpha}$ and $\widetilde{\operatorname{Inf} \beta}$ on the twisted crossed products. But the twisted crossed product $B \times{ }_{\operatorname{Inf} \beta, N} G$ is isomorphic to the ordinary crossed product $B \times{ }_{\beta} G / N$, and $\widetilde{\widehat{\operatorname{Inf} \beta}}$ corresponds to the dual coaction $\widehat{\beta}$ under this isomorphism. Consequently, $\widetilde{\alpha}$ is Morita-Rieffel equivalent to $\widehat{\beta}$; since $\widehat{\beta}$ is maximal, so is $\widetilde{\alpha}$. 
Corollary 7.2. Let $(A, G, \delta)$ be a maximal coaction, and let $N$ be a closed normal subgroup of $G$. Then the restricted coaction $(A, G / N, \delta \mid)$ is also maximal.

Proof. By maximality, $\delta$ is Morita-Rieffel equivalent (via the Katayama bimodule of Proposition 4.2 to a dual coaction $\left(B \times{ }_{\alpha} G, \widehat{\alpha}\right)$; hence the restricted coactions $\delta \mid$ and $\widehat{\alpha} \mid$ are also Morita-Rieffel equivalent. By Green's Decomposition Theorem 8 Proposition 1], the ordinary crossed product $B \times{ }_{\alpha} G$ is isomorphic to the twisted crossed product $B \times{ }_{\alpha \mid} N \times_{\alpha^{\mathrm{dec}}, N} G$, and it is easy to see that, under this isomorphism, $\widehat{\alpha} \mid$ corresponds to $\widetilde{\alpha^{\mathrm{dec}}}$, which is maximal by Proposition 7.1.

Proposition 7.3. Let $(A, H, \delta)$ be a maximal coaction, where $H$ is a closed subgroup of a locally compact group $G$. Then the inflated coaction $(A, G, \operatorname{Inf} \delta)$ is also maximal.

Proof. By maximality, $\delta$ is Morita-Rieffel equivalent (via the Katayama bimodule of Proposition 4.2) to a dual coaction $\left(B \times{ }_{\alpha} H, \widehat{\alpha}\right)$; hence the inflated coactions $\operatorname{Inf} \delta$ and $\operatorname{Inf} \widehat{\alpha}$ of $G$ are also Morita-Rieffel equivalent. By Green's Imprimitivity Theorem for induced algebras [8, Theorem 17], $B \times{ }_{\alpha} H$ is Morita-Rieffel equivalent to the crossed product $\operatorname{Ind}_{H}^{G} A \times_{\operatorname{Ind} \alpha} G$ by the induced action, and it is easy to see that the inflated coaction $\operatorname{Inf} \widehat{\alpha}$ corresponds to the dual coaction $\widehat{\operatorname{Ind} \alpha}$ under this isomorphism.

Proposition 7.4. Let $(A, G, \delta)$ be a maximal coaction, and let $N$ be a closed normal subgroup of $G$. Then the decomposition coaction $\left(A \times_{\delta \mid} G / N, G, \delta^{\text {dec }}\right)$ is also maximal.

Proof. By maximality, $\delta^{\text {dec }}$ is Morita-Rieffel equivalent (via the Mansfield bimodule of Theorem 5.3) to Inf $\widehat{\widehat{\delta}} \mid$, which is maximal by Proposition 7.3 .

Let $(B, G, \epsilon)$ be a coaction. A (closed two-sided) ideal $J$ of $B$ is invariant under $\epsilon$ in the sense of 13 if

$$
\overline{\epsilon(J)\left(1 \otimes C^{*}(G)\right)}=J \otimes C^{*}(G) ;
$$

Nilsen has shown [13, Proposition 2.1] that when this is the case, the restriction $\left.\epsilon\right|_{J}$ is a coaction of $G$ on $J$.

Proposition 7.5. Let $(B, G, \epsilon)$ is a maximal coaction, and let $J$ be an ideal of $B$ which is invariant under $\epsilon$ in the above sense. Then the restriction $\left(J, G,\left.\epsilon\right|_{J}\right)$ is also maximal.

Proof. Let $(\pi, \mu)$ be the canonical covariant homomorphism of $\left(B, C_{0}(G)\right)$ into $M\left(B \times{ }_{\epsilon} G\right)$; then by [13, Proposition 2.1], $\left.\pi\right|_{J} \times \mu$ is an isomorphism of $J \times_{\left.\epsilon\right|_{J}} G$ onto an ideal of $B \times{ }_{\epsilon} G$. 2 Identifying $J \times\left.{ }_{\epsilon}\right|_{J} G$ with its image in $B \times{ }_{\epsilon} G$, it is easy to see that this ideal is invariant under the dual action $\widehat{\epsilon}$. Thus by 8 , Proposition 12], the double crossed product $J \times_{\epsilon \mid J} G \times \times_{\left.\widehat{\epsilon}\right|_{J}} G$ can be identified with an ideal of $B \times{ }_{\epsilon} G \times \times_{\hat{\epsilon}} G$. But subject to these identifications, the canonical surjection $\Phi_{J}: J \times_{\left.\epsilon\right|_{J}} G \times \times_{\left.\widehat{\epsilon}\right|_{J}} G \rightarrow J \otimes \mathcal{K}$ is just the restriction of the isomorphism $\Phi_{B}: B \times_{\epsilon} G \times_{\widehat{\epsilon}} G \rightarrow B \otimes \mathcal{K}$, and hence is 1-1.

\footnotetext{
${ }^{2}$ There is a subtlety concerning how to make an "official" covariant homomorphism out of $\left(\left.\pi\right|_{J}, \mu\right)$, but Nilsen shows that this causes no problem.
} 
Associated to a right-Hilbert bimodule coaction ${ }_{(A, \delta)}(X, \zeta)_{(B, \epsilon)}$, there can be as many as three other $C^{*}$-coactions: first, there is a coaction $\mu$ on the imprimitivity algebra $\mathcal{K}_{B}(X)$ such that $\zeta$ is $\mu-\epsilon$ compatible, by [4, Proposition 2.30]; second, there is a coaction $\nu$ on the linking algebra $L(X)$ which compresses on the corners to $\mu, \zeta$, and $\epsilon$, by [4. Lemma 2.22]; third, the closed span $B_{X}=\overline{\langle X, X\rangle_{B}}$ is an $\epsilon$-invariant ideal of $B$, and the restriction of $\epsilon$ to $B_{X}$ is a coaction $\vartheta$ on $B_{X}$ such that $\zeta$ is $\mu-\vartheta$ compatible, by [4, Lemma 2.32].

Corollary 7.6. With notation as above, if the coaction $(B, G, \epsilon)$ is maximal, then so are $\left(\mathcal{K}_{B}(X), G, \mu\right),(L(X), G, \nu)$, and $\left(B_{X}, G, \vartheta\right)$.

Proof. Since $\mu, \nu$, and $\vartheta$ are all Morita-Rieffel equivalent, it suffices to show that $\vartheta$ is maximal; but this is immediate from Proposition 7.5.

\section{REFERENCES}

[1] S. Echterhoff, Morita equivalent twisted actions and a new version of the Packer-Raeburn stabilization trick, J. London Math. Soc. 50 (1994), 170-186. MF.96a:46118

[2] S. Echterhoff, S. Kaliszewski, and J. Quigg, Maximal coactions, Internat. J. Math. 15 (2004), 47-61. MF 2004j:46087

[3] S. Echterhoff, S. Kaliszewski, J. Quigg, and I. Raeburn, Naturality and induced representations, Bull. Austral. Math. Soc. 61 (2000), 415-438. MR2001j:46101

[4] _ A Categorical Approach to Imprimitivity Theorems for $C^{*}$-Dynamical Systems, preprint, 2002.

[5] S. Echterhoff, S. Kaliszewski, and I. Raeburn, Crossed products by dual coactions of groups and homogeneous spaces, J. Operator Theory 39 (1998), 151-176. MR99h:46124

[6] S. Echterhoff and J. Quigg, Full duality for coactions of discrete groups, Math. Scand. 90 (2002), 267-288. MR2003g:46079

[7] S. Echterhoff and I. Raeburn, The stabilisation trick for coactions, J. reine angew. Math. 470 (1996), 181-215. MR98c:46142

[8] P. Green, The local structure of twisted covariance algebras, Acta Math. 140 (1978), 191-250. MR.58:12376

[9] S. Kaliszewski and J. Quigg, Imprimitivity for $C^{*}$-coactions of non-amenable groups, Math. Proc. Cambridge Philos. Soc. 123 (1998), 101-118. MR99a:46118

[10] G. W. Mackey, Imprimitivity for representations of locally compact groups. I, Proc. Natl. Acad. Sci. USA 35 (1949), 537-545. MR 11:158b

[11] K. Mansfield, Induced representations of crossed products by coactions, J. Funct. Anal. 97 (1991), 112-161. MR92h:46095

[12] M. Nilsen, Duality for full crossed products of $C^{*}$-algebras by non-amenable groups, Proc. Amer. Math. Soc. 126 (1998), 2969-2978. MF99a:46120

[13] Math. Soc. 31 (1999), 556-568. MR.2000i:46065

[14] J. Quigg, Full and reduced $C^{*}$-coactions, Math. Proc. Cambridge Philos. Soc. 116 (1994), 435-450. MR95g:46126

Department of Mathematics and Statistics, Arizona State University, Tempe, AriZONA 85287

E-mail address: kaliszewski@asu.edu

Department of Mathematics and Statistics, Arizona State University, Tempe, AriZONA 85287

E-mail address: quigg@math.asu.edu 